\title{
L'écriture entre mémoire et oubli. Hommage à Krystyna Kasprzyk, W. Kroker et A. Sobczyk (éds.)
}

\section{Francesca Forcolin}

\section{(2) OpenEdition}

1 Journals

\section{Edizione digitale}

URL: http://journals.openedition.org/studifrancesi/10095

DOI: 10.4000/studifrancesi. 10095

ISSN: 2421-5856

\section{Editore}

Rosenberg \& Sellier

\section{Edizione cartacea}

Data di pubblicazione: 1 août 2017

Paginazione: $417-418$

ISSN: 0039-2944

\section{Notizia bibliografica digitale}

Francesca Forcolin, «L'écriture entre mémoire et oubli. Hommage à Krystyna Kasprzyk, W. Kroker et A. Sobczyk (éds.)», Studi Francesi [Online], 182 (LXI | II) | 2017, online dal 01 août 2017, consultato il 06 janvier 2021. URL: http://journals.openedition.org/studifrancesi/10095 ; DOI: https://doi.org/10.4000/ studifrancesi.10095

Questo documento è stato generato automaticamente il 6 janvier 2021.

\section{(c) (1)}

Studi Francesi è distribuita con Licenza Creative Commons Attribuzione - Non commerciale - Non opere derivate 4.0 Internazionale. 


\title{
L'écriture entre mémoire et oubli. Hommage à Krystyna Kasprzyk, W. Kroker et A. Sobczyk (éds.)
}

\author{
Francesca Forcolin
}

\section{NOTIZIA}

L'écriture entre mémoire et oubli. Hommage à Krystyna Kasprzyk, textes réunis par Wieslaw KROKER et Agata SOBCZYK, Institut d'Etudes Romanes, Université de Varsovie, 2016, 217 pp.

Scrittura e memoria, memoria e oblio, tre termini indissociabili: la scrittura permette di conservare la parola e il pensiero, ma permette anche l'esistenza di quei vuoti di memoria intorno ai quali si costruiscono i ricordi. La memoria individuale e collettiva, la costruzione del passato sulle tracce di quello che è stato e in vista di ciò che potrebbe perdersi è il filo conduttore dei quattordici contributi che compongono la miscellanea, ma è anche nel cuore stesso del volume, che si presenta come omaggio e ricordo di una delle più eminenti studiose polacche di letteratura francese (in particolare del periodo rinascimentale) Krystyna Kasprzyk (L'ancienne littérature française - sa grande passion. Hommage à Krystyna Kasprzyk di Dorota SzELIGA, pp. 11-20), di cui «Studi Francesi» ospitò un contributo nel n. 100 (Marguerite de Navarre lecteur du Décaméron). L'articolazione della memoria e della dimenticanza all'interno della scrittura è sviluppata seguendo un ordine cronologico. Il volume è pertanto suddiviso in tre grandi parti, preceduti dall'Avant-propos di Wieslaw KROKER (pp. 7-10): «La mémoire et les pouvoirs symboliques sous l'Ancien Régime»; «La mémoire individuelle et l'image sociale de soi aux XVIII et $\mathrm{XIX}^{\mathrm{e}}$ siècles»; e «Le long $\mathrm{XX}^{\mathrm{e}}$ siècle: la lutte contre l'oubli entre l'intime et le politique». Come ricorda Maciej ABRAMOWICZ nel primo contributo, La fausse mémoire et l'axiologie chevaleresque (pp. 21-36), all'epoca medievale il modello della scrittura narrativa era fornito dalla storiografia: la scrittura, dunque, affonda le sue radici nella Storia che ne è 
il sostrato evidente, rapportando la realtà indiscussa dell'epoca, come le imprese di Carlomagno. Ma tale evidenza di realtà storica crolla con la nascita e la diffusione del romanzo e con la conseguente idealizzazione del passato che diviene l'espressione delle aspirazioni e non un riflesso della realtà sociale. Sempre sull'epoca medievale verte l'articolo successivo, L'écriture des "Miracles de Notre-Dame de Chartres" entre la mémoire et l'oubli (pp. 37-52) di Agata sовсZYK, che presenta un caso interessante di sovrapposizione di strati di memoria: nel passaggio dalla prosa latina di un autore anonimo alla sua traduzione francese in versi da parte di Jean le Marchant, i Miracles de Notre-Dame de Chartres vengono rinfoltiti da un numero maggiore di miracoli, di cui il traduttore aveva udito o letto testimonianza, che si erano manifestati a Chartres in seguito all'incendio che aveva distrutto la Chiesa ma salvato la reliquia della camicia di Notre-Dame: ciò attesta un passaggio dalla memoria individuale dell'autore latino alla memoria collettiva dei credenti. Con la studiosa pugliese dell'Università Aldo Moro di Bari Concetta CAVALLINI, si passa al Rinascimento: in "La Resjouissance sur la France désolée" de Benoît Voron (1574) entre mémoire et oubli (pp. 53-68), ci si sofferma sul dialogo in alessandrini di Voron che, attraverso due personaggi allegorici, Resjouissance e France, discute sulla società dell'epoca, tra lamentatio e speranze, mescolando la tradizione letteraria classica del didattismo e moralismo prossimi al teatro scolastico a elementi innovativi che, tuttavia, assicurano nulla di più di una testimonianza storica. Con Maja PAWLowsKa passiamo al 1664, anno della pubblicazione di La bibliothèque française di Sorel ("La bibliothèque française" de Charles Sorel et la formation de la mémoire historique de l'honnête homme, pp. 69-81), che permette di riflettere sulla concezione della formazione dell' «honnête homme»: opera enciclopedica, la Bibliothèque risponde al desiderio di salvare l'uomo di qualunque ceto sociale dall'ignoranza, colmando la necessità di erudizione attraverso l'organizzazione e la presentazione critica di un necessario bagaglio culturale.

2 La seconda sezione si apre con il contributo di Catriona SETH, Autoportrait d'outre-tombe: les "Mémoires" de Mary Robinson (pp. 83-96): nella sua opera autobiografica, Mary Robinson modella la propria immagine di attrice con l'obiettivo di difendere la propria reputazione e la propria fisicità e iscriversi in tal modo nella durata, affermandosi come individuo oltre le critiche e i pettegolezzi. Scritti personali ma radicati nella Storia sono, durante il secolo dei Lumi, due in particolare, come ricorda Regina BOCHENEKFRANCZAKOWA (Écrire pour soi et témoigner pour la postérité au temps de la Révolution française (J.-B. Louvet, L.-S. Mercier), pp. 97-108): Quelques notices pour l'histoire di Louvet e Nouveau Paris di Mercier, si caratterizzano per la mescolanza di elementi pubblici e privati. Gli autori, infatti, non raccontano soltanto le proprie vite, ma si raccontano come individui all'interno della Rivoluzione, partecipando e riportando gli avvenimenti dell'epoca e consegnando in tal modo opere di indubbio valore testimoniale. Un interessante contributo è quello di Damien zANONE, Continuité des parcs: l'art de la mémoire de Chateaubriand (pp. 109-120): partendo da un racconto di Cortazar, mise en abîme che si sviluppa interamente in un parco, Zanone ritrova questo locus amoenus nel libro VI dei Mémoires d'outre-tombe, dove le scene nei parchi si intrecciano ripetutamente al punto da poter essere identificate come un leitmotif che riporta senza tregua alla vita dello scrittore durante gli anni della sua giovinezza a Londra. Un tentativo, dunque, di recuperare il proprio passato, la propria storia individuale. Anna OPIELA-MROZIK in La mémoire de l'art, l'art de la mémoire. Delacroix et Berlioz à travers leurs écrits intimes (pp. 121-133), analizza il rapporto con il ricordo e la memoria nelle opere dei due artisti. Si 
tratta di pratiche opposte, pur in parallelo con la produzione artistica di entrambi: se Delacroix inizia a scrivere il Journal per fissare i progetti della pittura, le idee sull'arte per il timore che queste col tempo si perdano, Berlioz redige i Mémoires con l'intento di lasciare una traccia di sé e della propria musica, una maniera per difendersi e spiegarsi davanti alla posterità.

La terza e ultima sezione copre il xIx e il xx secolo. Kamil popowicz firma Un cas particulier de la mémoire: Arthur de Gobineau et son cuvre (pp. 135-146), stimolante contributo che permette di riflettere sulla memoria postuma di Gobineau (1816-1882): benché egli abbia scritto un'autobiografia, ciò che ci resta è soprattutto l'immagine di uno dei primi esponenti del razzismo, colui che seguì e fece proprie le teorie preevoluzioniste di Buffon, sviluppate poi con Darwin e riprese con efferato orrore dall'ideologia nazista. Julia LUKASIAK, con L'image de la mort: entre la mémoire et l'oubli. L'exemple de "Dulle Griet" de Dominique Rolin (pp. 147-158), riflette invece sul complesso lavoro Dulle Griet, opera al limite tra memoria e oblio del padre scomparso, il cui ritratto si intesse con la descrizione dell'omonimo quadro di Bruegel (1561), rappresentazione fantastica e ricca di riferimenti alchemici della leggenda di Margherita d'Antiochia, come se il quadro fosse il prolungamento o il doppio del genitore. Più teorico è invece il breve articolo di Miroslaw LOBA, Oublier selon Roland Barthes (pp. 159-166), analisi del postumo e incompiuto Journal de deuil: riflessioni, appunti, testimonianza sulla separazione dalla madre dopo la sua morte, dove la scrittura si fa strumento per recuperare e riabilitare l'oggetto perduto. Complesso è La dystopie comme fiction de l'oubli (George Orwell, Paul Auster, Tatiana Tolstoï, Lutz Bassman, Michel Houellebecq) di Anna SAIGNES (pp. 167-180), riflessione sulla distopia intesa come opera che pensa il ruolo della memoria e della dimenticanza nel funzionamento di una data società, collegando tali concetti con il potere, i legami sociali e la costruzione di un'identità nazionale. In particolare, vengono brevemente esaminati 1984 di Orwell, In the country of last things di Auster, Kys di Tolstoï, Avec les moins soldats di Bassman, Les particules élémentaires di Houellebecq. In questo percorso non poteva mancare una riflessione su Modiano, firmata da Wieslaw KROKER (Le silence et les voix entre mémoire et oubli dans "Vestiaire de l'enfance" de Patrick Modiano, pp. 181-200), in cui il testo preso in esame di Modiano è presentato come un esempio di commistione di memoria personale (l'infanzia, l'assenza dei genitori, la solitudine, le tracce del passato attraverso sensazioni olfattive e visive) e collettiva (le zone d'ombra del periodo dell'Occupazione nazista, l'ebraismo). Chiude il volume l'indice degli autori uniti nel ricordo di Krystyna Kasprzyk. 